\title{
Evidence of Somatic Pairing in an Adenocarcinoma of the Lung
}

\author{
S. A. Faruqi ${ }^{1}$, J. S. Noumoff ${ }^{1}$ and L. J. Krueger ${ }^{2}$ \\ ${ }^{1}$ Department of OB/Gyn, Oncology Division, Crozer-Chester Medical Center, Upland, PA 19013, USA \\ ${ }^{2}$ Department of Surgery, St. Christopher Hospital for Children, Eiry Ave, on Front Street, Philadelphia, PA 19134, USA
}

Accepted December 14, 1998

\begin{abstract}
Summary Chromosomal analysis of an adenocarcinoma of the lung showed three clones. Additionally three cells were also recorded which showed mitotic chromosomal pairing involving varying number of chromosomes. In one of these cells almost the entire genome was involved in mitotic pairing of chromosomes. The importance of somatic pairing of chromosomes in the context of tumor evolution is highlighted.
\end{abstract}

During the last decade, lung cancer (CA) has become the leading cause of deaths by cancer in both men and women. In the United States, lung cancer has an annual incidence of some 180,000 cases with approximately 150,000 deaths. Unlike hematologic neoplasms where chromosomal aberrations correlate with diagnosis, management and prognosis, similar information regarding solid tumors is not yet recognized.

We have made the observation in a case of adenocarcinoma of the lung which showed three different clones and also mitotic pairing of GTG banded homologous chromosomes. We have highlighted the significance of mitotic pairing of chromosomes in tumor evolution.

\section{Material and method}

After excision, the tumor diagnosed as adenocarcinoma of the lung was brought in RPMI1640 immediately to the laboratory. It was minced and exposed to $16 \mathrm{mg}$ of collagenase in $10 \mathrm{ml}$ RPMI-1640. Cultures were established in slide-chambers in RPMI-1640 supplemented with 10\% FCS and $1 \%$ each of L-glutamine and Pen/Strep and cells were harvested in-situ after $0.04 / \mathrm{mg}$ colchecine exposure for $1 \mathrm{hr}$. Chromosomes were banded after trypsin treatment and stained in Giemsa for GTG banding. Twenty cells were analyzed for chromosomal aberrations.

\section{Results}

GTG banded metaphase chromosomal analysis of an adenocarcinoma of the lung showed the presence of three clones with the following karyotypes: 45,X[7]/47,XY, + mar[2]/46,XY[11]. In addition to these clones, three cells in close proximity to each other showing somatic pairing among homologous chromosomes were observed on one of the slides. Chiasmata were absent in each of the chromosomal pairs. The cell with the maximum number of pairs showed chromosomal association of almost the entire genome (Fig. 1). 


\section{Discussion}

Mitotic chromosomal pairing and chiasma formation is known to have existed in different organisms, yet its genetic consequence in the origin of cancers and other genetic diseases was late to be recognized. Radman and Kinsella (1980) were the first to point-out the significance of somatic recombination in the origin of homozygosity of a heterozygous allele. Gregory et al. (1991) proposed a model explaining somatic recombination in their study of Prader-Willi syndrome and Woodage et al. (1994) in Bloom syndrome. Faruqi et al. (1994) on the basis of their studies on normal amniotic cells and blood, demonstrated not only mitotic pairing and chiasma formation but also provided the first objective proof of chro-

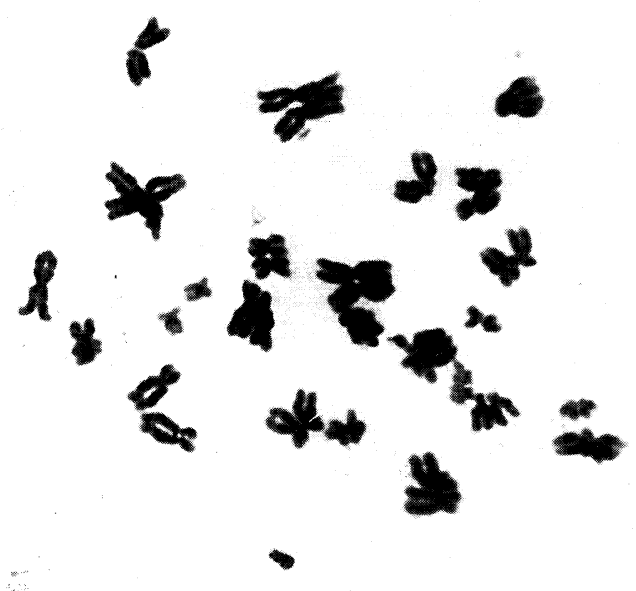

Fig. 1. A diploid cell from an adenocarcinoma of the lung, showing somatic pairing of almost the entire genome. matid exchange and consequently validity to the alternate method of loss of heterozygosity rather than mutation in the etiology of cancers and other genetic diseases controlled by Mendelian inheritance of recessive alleles.

We present mitotic chromosomal pairing in an adenocarcinoma of the lung and propose that this mechanism may be playing a much more significant role in the etiology and evolution of tumors than it is realized todate.

\section{References}

Faruqi, S. A., Miller, R. C. and Noumoff, J. S. 1994. Somatic pairing: An alternative for the development of cancer and other hereditary diseases. Cytologia 59: 439-444.

Gregory, C. A., Schwartz, J., Kirkilionis, A. J., Rudd, N. and Hamerton, J. L. 1991. Somatic recombination rather than uniparental disomy suggested as another mechanism by which genetic imprinting may play a role in the etiology of Prader-Willi syndrome. Hum. Genet. 88: 42-48.

Radman, M. and Kinsella, A. R. 1980. Chromosomal events in carcinogenic initiation and promotion: implications on carcinogenicity and cancer prevention strategies. IARC Scientific Publications 27: 75-90.

Woodage, T., Prasad, M., Dixon, J. W., Selby, R. E., Romain, D. R., Columbano-Green, L. M., Graham, D., Rogan, P. K., Seip, J. R., Smith, A. and Trent, R. J. 1994. Bloom syndrome and maternal uniparental disomy for chromosome 15. Amer. J. Hum. Genet. 55: 74-80. 